Scott, L. J., M. SHEPHERD and R. J. HenRy (2003): Characterisation of highly conserved microsatellite loci in Araucaria cunninghamii and related species. Plant Systematics and Evolution 236: 115-123.

Van EijK, M., M. De Ruiter, J. Broekhof and J. Peleman (2001): Discovery and detection of polymorphic microsatellites by microsatellite-AFLP, pp 143. In: Plant and animal genome IX abstracts, edited by S. R. HELLER, Scherago International, New York.

Van Oosterhout, C., W. F. Hutchinson, D. P. M. Wills and P. SHIPLEY (2004): Micro-Checker: software for identifying and correcting genotyping errors in microsatellite data. Molecular Ecology Notes 4: 535-538.
Veblen, T. T. (1982): Regeneration patterns in Araucaria araucana forest in Chile. Journal of Biogeography 9: 11-28.

Vos, P., R. Hogers, M. Sleeker, M. Reijans, T. Lee and M. Homes et al. (1995): AFLP: a new concept for DNA fingerprinting. Nucleic Acids Research 23: 4407-4414.

Yang, H., M. W. Sweetingham, W. A. Cowling and P. M. C. SMITH (2001): DNA fingerprinting based on microsatellite-anchored fragment length polymorphisms, and isolation of sequence-specific PCR markers in lupin (Lupinus angustifolius L.), Molecular Breeding 7: 203-209.

\title{
Transcript abundances of LIM transcription factor, 4CL, CAld5H and CesAs affect wood properties in Eucalyptus globulus
}

\author{
By N. NeGiShI ${ }^{1)}$, K. NANTO ${ }^{1)}$, K. HAYAShI ${ }^{1)}$, S. ONOGI ${ }^{1)}$ and A. KAWAOKA ${ }^{\left.1),{ }^{*}\right)}$
}

(Received 21 ${ }^{\text {th }}$ July 2011)

\begin{abstract}
Eucalyptus globulus is the main hardwood species grown in pulpwood plantations in temperate regions of the world. We have cloned six genes influencing wood quality including the LIM domain transcription factor (LIM), 4-coumarate-CoA ligase (4CL), coniferaldehyde 5hydroxylase $(\mathrm{CAld} 5 \mathrm{H})$ and the three catalytic units of cellulose synthase (CesA), from $E$. globulus. The transcript abundances of LIM in basal stems of ten independent $E$. globulus lines showed similar patterns to those of $4 \mathrm{CL}$. We investigated the correlation between gene transcript abundances and wood qualities such as Klason lignin (KL) content, syringaldehyde/vanillin (S/V) ratio and holocellulose (HC) content. Expression of the LIM and 4CL were positively correlated with KL content. A highly significant positive correlation was observed between $\mathrm{CAld} 5 \mathrm{H}$ expression and $\mathrm{S} / \mathrm{V}$ ratio. Furthermore, a ratio of the sum of the transcript abundances of three CesA1, CesA2 and CesA3 to 4CL showed a positive correlation with a ratio of $\mathrm{HC} / \mathrm{KL}$ content that positively correlated with the chemically extracted fiber content in this woody plant.
\end{abstract}

Key words: LIM domain transcription factor, CAld5H, CesA, 4CL, Eucalyptus globulus.

\section{Introduction}

Eucalyptus species constitute the most widely planted hardwood trees in temperate and subtropical regions.

\footnotetext{
1) Agri-Biotechnology Research Laboratory, Nippon Paper Industries Co. Ltd. 5-21-1 Oji, Kita-ku, Tokyo 114-0002, Japan.

*) Authors for further correspondence: AkIYOSHI KAWAOKA. Phone 81-3-3911-3084; Fax 81-3-5902-4782.

E-Mail: akawaoka@np-g.com
}

Their wood is used as a raw material for the production of cellulose. Eucalyptus species have fast growth rates and the ability to adapt to a broad range of geographic locations. Eucalyptus has been listed as one of the candidate biomass energy crops (LI et al., 2008; HINCHEE et al., 2009). Eucalyptus globulus is one of the most important commercial temperate hardwood species for the pulp and paper industries because of its wood properties and pulp production characteristics.

Plant cell walls are composed mostly of cellulose, hemicellulose and lignin. Cellulose provides strength and flexibility to plant tissue and is of great importance to the chemical, textile, pulp and paper industries. In woody plants, high levels of cellulose are synthesized and cellulose accounts for about $50 \%$ of the dry weight of wood. However, the precise biosynthetic pathway of cellulose is not well understood, although genome-level studies are underway in some model plants such as Arabidopsis thaliana and Populus trichocarpa (ENDLER and Persson, 2011; KumAR et al., 2009). Most structural genes involved in lignin biosynthesis have been identified in many species including woody plants (BOERJAN et al., 2003). The aromatic lignin polymers commonly found in woody plants are primarily composed of two monolignols, namely coniferyl and sinapyl alcohols, which typically form guaiacyl-syringyl (G-S) lignin when polymerized. These monolignols are synthesized via the phenylpropanoid pathway, which begins with deamination of phenylalanine to form cinnamate, followed by a series of ring hydroxylations, $O$-methylations, and sidechain modifications. Lignin found in gymnosperms and ferns lack S units (BOERJAN et al., 2003) suggesting that the branch leading to sinapyl alcohol biosynthesis may 
be a relatively recent addition to the angiosperms' biochemical repertoire. Evolutionally, Selaginella, a primitive plant forms S-lignin in vascular tissue (WENG et al., 2008). Our understanding of the monolignol biosynthetic pathway has undergone multiple major revisions over the past decade due to the results generated from both in vitro kinetic studies on lignin biosynthetic enzymes and from genetic studies on mutants and transgenic plants with altered transcript abundances of phenylpropanoid pathway genes (BOERJAN et al., 2003; Humphreys and CHAPPle, 2002). Current thinking suggests that ten enzymes are required for monolignol biosynthesis. In the cell wall, peroxidases and laccases are believed to catalyze the dehydrogenative polymerization of the monolignols (BOERJAN et al., 2003).

A report by LI et al. (2003) concerning lignin content and composition indicated that transgenic aspen plants exhibiting downregulation of 4CL and upregulation of $\mathrm{CAld5H}$ had lowered lignin content and an increased $\mathrm{S} / \mathrm{G}$ ratio. In angiosperm trees, lignin is polymerized from $\mathrm{G}$ and $\mathrm{S}$ monolignols and the $\mathrm{S}$ unit is thought to be more reactive to chemical hydrolysis than the $\mathrm{G}$ unit (SARKNEN, 1971). A low lignin content and high S/G ratio in plant cell walls are desirable factors to improve the pulp production efficiency. $4 \mathrm{CL}$ and $\mathrm{CAld} 5 \mathrm{H}$ are the key enzymes that control the lignin content and S/G ratios. Master genes in the regulatory network of secondary wall formation, such as the NAC domain transcription factor, have been identified (KUBO et al., 2005; MITSUDA et al., 2007). The involvement of several other transcription factors in lignin biosynthesis has also been reported (Rogers and CAMPBell, 2004; Goicoechae et al., 2005). One of these, the LIM domain transcription factor (LIM), binds specifically to a PAL-box element, thought to be an important cis-acting element of lignificationrelated gene expression (KAWAOKA et al., 2000; KAOTHIEN et al., 2002). Transgenic tobacco and Eucalyptus camaldulensis with antisense LIM show low concentrations of transcripts of some key genes such as $P A L$ and $4 C L$ that are involved in the lignin biosynthesis pathway (KAWAOKA et al., 2000; KAWAOKA et al., 2006). Thus, LIM plays a crucial role in lignification. Another gene involved in lignin biosynthesis is the coniferaldehyde 5hydroxylase $(\mathrm{CAld} 5 \mathrm{H})$. This gene, along with $4 \mathrm{CL}$, has been implicated in wood formation of $E$. gunnii (RENGEL et al., 2009).

Other key genes implicated in wood quality are the cellulose synthase (CesA) genes. CesA was first cloned from cotton fibres as a plant homologue of bacterial cellulose synthase (PEAR et al., 1996). To date, 10 CesA protein encoding genes have been identified in the genome of Arabidopsis and of these six CesA proteins have been associated with CesA complexes active during primary wall formation (ENDLER and PERSSON, 2011). The other four CesA have been reported to be part of the CesA complex responsible for secondary wall cellulose synthesis (ENDLER and PERSSON, 2011). In E. grandis, a total of seven $C e s A$ genes are expressed and three CesA genes (referred to as CesA1 to 3) exhibit elevated transcript abundance during secondary wall formation (RANIC and Myburg, 2006). Gallo DE CALvalho et al. (2008) also observed similar transcriptional profiles by serial analysis of gene expression (SAGE). The specific role of each CesA component in the overall cellulose synthase pathway remains obscure.

In this study, we focused on above six key genes involved in wood formation, including those encoding the LIM domain transcription factor, the enzymes 4CL, $\mathrm{CAld} 5 \mathrm{H}$, and three CesA genes from E. globulus. We investigated their transcript levels and the relationships between their expression and factors determining wood quality such as Klason lignin content (KL), syringaldehyde/vanillin $(\mathrm{S} / \mathrm{V})$ ratio and holocellulose $(\mathrm{HC})$ content. We found that the transcript abundances of LIM, 4CL, and CAld5H were highly correlated with lignin content and/or composition in the basal part of stems. Furthermore, we also predict that gene expressions of the three CesAs and 4CL relate to the fiber content which is chemically extracted in this woody plant.

\section{Materials and Methods}

\section{Plant material}

$E$. globulus plants for this study were selected on the plantation of Bunbury Treefarm Project, Nippon Paper Resources, Western Australia. The clonal E. globulus plants were produced by a photoautotropic culture method (NAGAE et al., 1996) and were grown in a greenhouse using culture pots (diameter $45 \mathrm{~cm}$, depth $45 \mathrm{~cm}$ ) under natural photoperiod (12.5-14.6 hours of daylength). Each plant tissue was collected at September from six-month old plant grown in the greenhouse.

\section{Isolation of LIM, 4CL CAld5H and CesA genes}

A cDNA library was constructed using mRNA purified from young stems of six-month old $E$. globulus grown in the greenhouse. The cDNAs were screened by plaque hybridization using DNA probes prepared by PCR according to the nucleotide sequence information from $E$. grandis and $E$. gunnii. The inserted DNA fragments of positive clones were amplified by PCR using forward and reverse primers for the $\lambda$ ZAPII vector (Stratagene, La Jolla, CA, USA). Sequencing was carried out using a Beckmann XL2000 sequencer (Fullerton, CA, USA). Primers (Table 1) were designed for amplification each genomic DNA and cDNA.

Table 1. - Gene-specific primers used for amplification cDNA and genomic DNA.

\begin{tabular}{|c|c|c|}
\hline & Forward & Reverse \\
\hline Eg|LIM1 & GGCTTCCCTTTCTTATCCTCCATTCT & GGACAAGACTGAAAAGAAAGCAAGCTCAGA \\
\hline Eg|4CL1 & GTCTCTCGATTCTCCGCCCCGCCACGACAA & TGAAAAGAAAGGGTATGAGAAATTAATTGT \\
\hline EglCAld5H1 & TCCTCTCCAAAAGAGCTAATCCATGGATAT & TTCTTCATTCCATATTTATTTGTGTATGAC \\
\hline Eg|CesA1 & GTTGGCAATTGGACTTCTGAGGTATTGCTC & AGTAACATAATAACACCCTTGCTTTGACCA \\
\hline Eg|CesA2 & GCGCGCCTTCCCCTCGCGATCGTTTCCCGC & ATGTCCCAAGTAAGAGACAGAAAGAAACAG \\
\hline EglCesA3 & CCATGGAAGCCGGAGCTGGACTTGTCGCCG & GAAATGTAGGATTGATTTTTTTTTAGTATGA \\
\hline
\end{tabular}


Table 2. - Gene-specific primers used in quantitative RT-qPCR.

\begin{tabular}{|l|l|l|}
\hline \multicolumn{2}{|l|}{ Forward } & Reverse \\
\hline Eg|LIM1 & GGGAACTATAATTCATTTGAAGGAG & AGTTGGCTGAGATTGCCCTTCTCCTT \\
\hline Eg|4CL1 & CGTTGTTCCACATATACTCCCTCAAC & GCGGTCGCTTCGGGGTCGTTCAGA \\
\hline Eg|CA|d5H1 & TCCGCAAGGGCTCACCGCCAGGCT & ACCAACCTCGGAGTCGGCACCGCCA \\
\hline Eg|CesA1 & AGGGAAGACCTAAATGCCGCCATC & GGGCATGCAATAGACCGACCTCCA \\
\hline Eg|CesA2 & TCAGAATGGTCAGGTTTTCTCTTC & TCCTTCACAAAGCTGGGCTCCACC \\
\hline Eg|CesA3 & GGGCTGGATTTATGGTTCTATCA & GTAGGATTGATTTTTTTTAGTATGA \\
\hline Eg|UBI & CCAAGATCCAGGACAAGGAA & CCTCGAGCTTAATGGTCTCG \\
\hline
\end{tabular}

\section{Quantitative Real-time (RT-q) PCR}

RT-qPCR was carried out on cDNA the total RNA from each organ. Total RNAs were extracted from shoot tips, young stems at $15-20 \mathrm{~cm}$ from the top, middle stems at about $50 \mathrm{~cm}$ from top, base stems at $10 \mathrm{~cm}$ from bottom, leaves and roots of 6-month old $E$. globulus (height: 1.0 $\mathrm{m})$ grown in a greenhouse (CHOMCZYNSKI and SACCHI, 1987). Primers for RT-qPCR (Table 2) were designed with Primer 3 (http://frodo.wi.mit.edu/primer3/). The cDNA templates were diluted 500 times prior to amplification. RT-qPCR experiments were carried out in an MJ Real-time PCR system Opticon2 (Bio-Rad, Hercules, CA, USA). A $15 \mu$ aliquot of a master mix consisting of $10 \mu \mathrm{l}$ of 2x SYBR Green JumpStart Taq Ready Mix (Sigma, St. Louis, MO, USA), $0.5 \mu \mathrm{l}$ each of $20 \mu \mathrm{M}$ forward and reverse oligonucleotides corresponding to a given target gene, and $4 \mu \mathrm{l}$ PCR-grade water was dispensed into 96well plates. The templates $(5 \mu \mathrm{l})$ were then added to the master mixes and transferred to the thermal cycler. Cycling conditions were 5 min of denaturation at $95^{\circ} \mathrm{C}$ followed by 40 cycles of $95^{\circ} \mathrm{C}$ denaturation for $15 \mathrm{~s}, 60^{\circ} \mathrm{C}$ annealing for $15 \mathrm{~s}$ and $72^{\circ} \mathrm{C}$ elongation for $20 \mathrm{~s}$. Following amplification, a dissociation analysis was carried out to detect any complex products. Data analysis was performed with RQ manager software (Applied Biosystems, Foster city, CA, USA) and transcript abundance was determined relative to the $E$. globulus ubiquitin gene as an internal standard. The program CORREL was used for a correlation analysis.

\section{Cell wall composition analysis}

Lignin determination was performed on the dried insoluble cell wall residues (CWR) of samples Soxhlet extracted with toluene/ethanol (1/2 [v/v]), ethanol and water. Klason lignin was measured by the method of EFFLAND (1977). To determine the monomeric composition of lignin in the CWR, we examined the CWR from each plant by alkaline nitrobenzene oxidation analysis. Vanillin and syringaldehyde are generated from the non-condensed fraction of lignin by this procedure (CHIANG and FunAOKA, 1988). Holocellulose was determined by sulfide acid extraction (WISE et al., 1946).

\section{Chemical Pulping}

Stems of the 7-10 year-old trees (stem volumes were $0.15-0.20 \mathrm{~m}^{3}$ ) grown in a eucalyptus plantation of Western Australia were harvested for chemical pulping analysis. Trees were debarked and cut into chips with a wood log chipper and screened to remove the coarse and fine elements with a chip size classifier. The kraft pulping process was simulated at laboratory scale on $200 \mathrm{~g}$ of oven-dried wood chips in small pressurized reactors in a rotating oil-thermostatic bath under the following conditions: active alkali: 14 to $16 \%$, sulfidity: $25 \%$, liquor/wood ratio: $2.5 / 1$, temperature was increased to $160^{\circ} \mathrm{C}$ over $90 \mathrm{~min}$, and maintained for $1 \mathrm{~h}$ for cooking. Pulps were washed and screened on a $150-\mu \mathrm{m}$ slotted screen to determine uncooked particles and pulp yield. Kappa numbers were determined according to international standards (NF ISO 302 and ISO 5351-1).

\section{Results}

\section{Gene structure of Eucalyptus globulus LIM, 4CL and CAld5H genes}

First, we isolated cDNAs of the LIM, 4CL, and CAld5H from $E$. globulus by hybridization of $\lambda$ ZAPII plaques and labeled probes. In $E$. grandis genome database (EucGenIE; http://eucgenie.org/), seven LIM transcription factors were registered. From the homology of amino acid sequence and the expression profile, a genome position Egrandis_v10.027973m.g was ortholog involved in lignin biosynthesis. The E. globulus LIM was identified using a cDNA fragment containing the coding region of E. camaldulensis EcLIM1 as a probe. The positively hybridized clone contained an open reading frame that encodes a protein of 188 amino acids and is identical with EcLIM1. We designated the protein encoded by this cDNA as EglLIM1. A 1.8-kbp genomic DNA fragment containing EglLIM1 was isolated by PCR using specific primers (accession numbers, EglLIM1 cDNA, AB208709; EglLIM1 genomic, AB208710). EgLIM1 contains five exons (Figure 1a). The deduced amino acid sequence of EglLIM1 was found to be highly similar to those of other plant LIM proteins described previously (KAWAOKA et al., 2006).

Next, we isolated the genes $4 C L$ and $C A l d 5 H$ of $E$. globulus in the same manner. In $E$. grandis genome database EucGenIE, four 4CL and seven CAld5H transcripts were listed, respectively. A fragment of $E$. gunnii $4 C L 1$ cDNA was used as a probe for the identification of the $4 C L$ cDNA (GALlO DE CALVALHO et al., 2008). A DNA fragment of the $E$. gunnii $C A l d 5 H$ gene was used for the isolation of the CAld5H cDNA, which is mostly expressed in xylem (RENGEL et al., 2009). Then, the Egl4CL1 and EglCAld5H1 genes were isolated and their nucleotide sequences were determined (accession numbers, Egl4CL1 cDNA, AB527047; Egl4CL1 genomic, AB368720; EglCAld5H1 cDNA, AB527050; EglCAld5H1 genomic, AB527049). The putative amino acid sequences of each gene showed high similarities with other plant gene homologues. Genomic DNA segments containing the Egl4CL1 and EglCAld5H1 genes were isolated by PCR using specific primers. The Egl4CL1 and 
EglCAld5H1 genes were found to contain five and two exons, respectively (Figure 1a).

Organ-specific expression of LIM, 4CL and CAld5H

Total RNA was extracted from shoot tips, young, middle and basal parts of stems, leaves and roots of E. globulus plants grown in a greenhouse. A young stem contain a cell-elongation region indicating a primary cell wall formation stage, while middle and basal parts of stem carry out secondary cell wall formation. The transcript levels of EglLIM1, Egl4CL1 and EglCAld5H1 in each organ were assayed by RT-qPCR (Figure 1b,c). The EglLIM1 and Egl4CL1 mRNAs were abundant in all organs studied, except for the leaves. The EglCAld5H1 transcript also accumulated in stems of all ages, but to a lesser extent in leaves and roots.

a

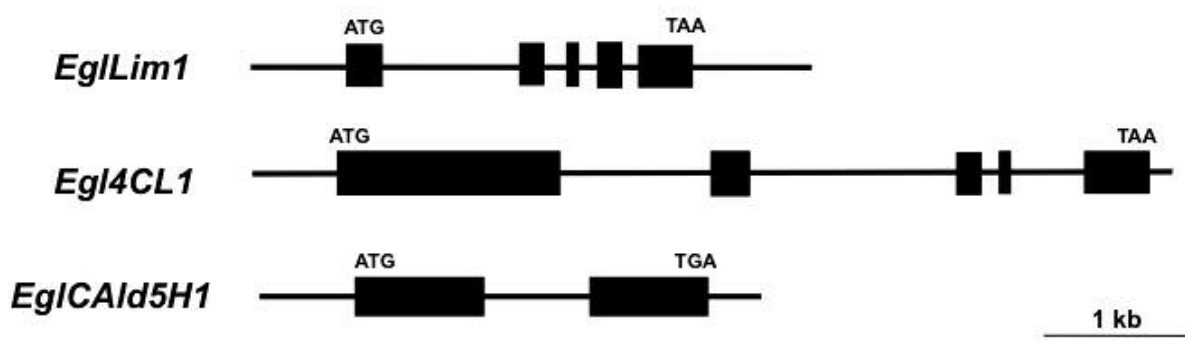

b

$\begin{array}{llllll}1 & 2 & 3 & 4 & 5 & 6\end{array}$

EgILim1

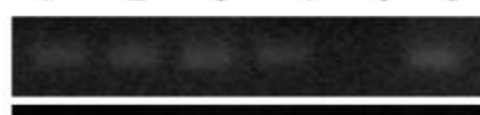

EgI4CL1

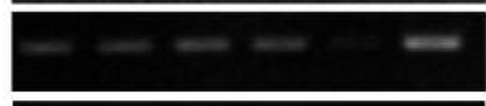

1. Shoot tip

EgICAId5H1

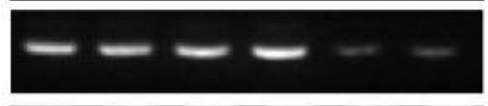

EgIUBI

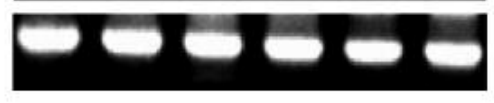

2. Young stem

3. Middle stem

4. Base stem

5. Leaf

6. Root

C
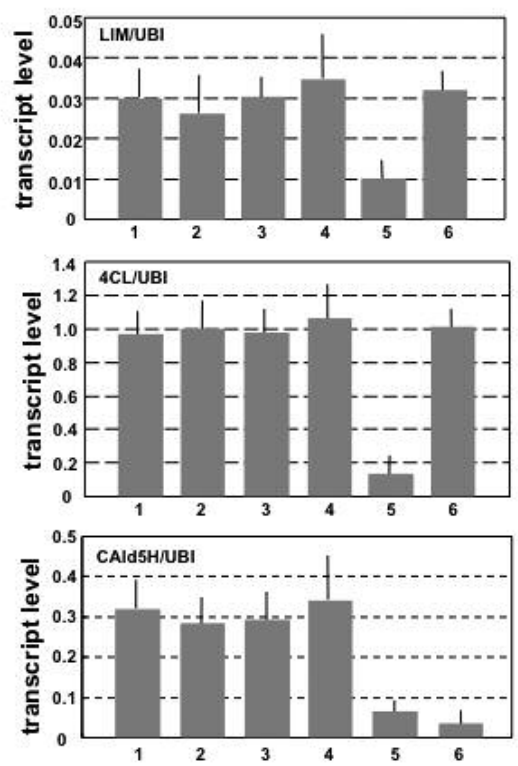

Figure 1. - Characterization of genes involved in E. globulus lignin biosynthesis.

a. Structure of the EglLIM1, Egl4CL1 and EglCAld5H1 genes. Rectangular boxes show the exons; lines between exons indicate introns.

b. RT-PCR gel analysis of EglLIM1, Egl4CL1 and EglCAld5H1 gene expressions in $E$. globulus. Total RNA was extracted from each organ.

c. RT-qPCR analysis of EglLIM1, Egl4CL1 and EglCAld5H1 gene expressions in E. globulus. Ubiquitin (EglUBI) gene was used as an internal control. Each blot was replicated at least three times with fresh plant extracts. Total RNAs were extracted from different organs viz. shoot tip (1), young stem (2), middle stem (3), base stem (4), leaf (5) and root (6). 


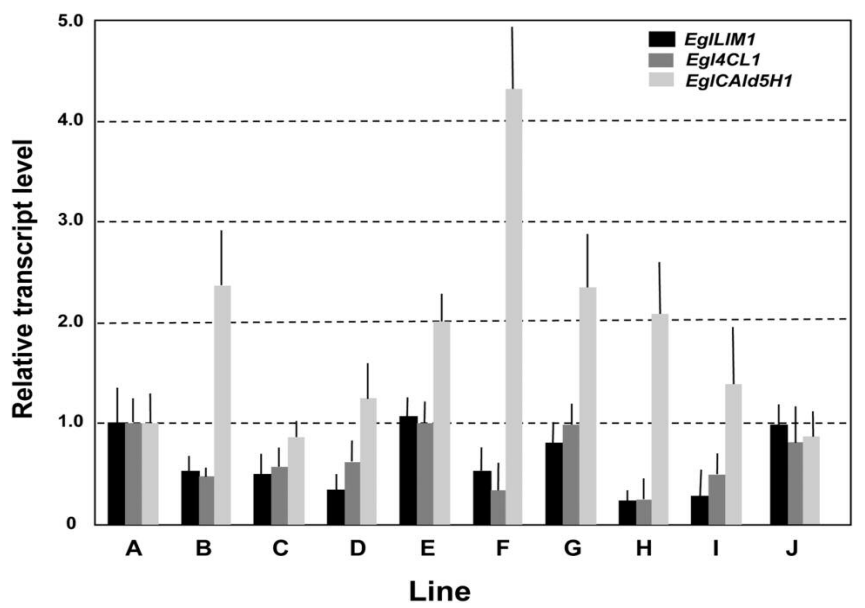

Figure 2. - Relative expression levels of the EglLIM1, Egl4CL1 and EglCAld5H1 genes. Total RNA was extracted from stems of ten independent lines and RT-qPCR was carried out using gene-specific primers. The Eucalyptus ubiquitin gene was used as an internal control. Each data point represents the average of three independent replicates and the error bars represent standard error values.
Transcript levels of EglLIM1, Egl4CL1and EglCAld5H1 in independent $E$. globulus lines

Previously, we reported that a LIM regulated the expression of PAL, C4H and 4CL (KAWAOKA et al., 2006). Therefore, transcript levels of LIM and 4CL were investigated in different $E$. globulus lines to find out if the transcript abundance is similar across different lines. Total RNAs were extracted from basal parts of the stems of 6-month old plants (about $1 \mathrm{~m}$ high) of ten $E$. globulus lines and RT-qPCR was carried out (Figure 2). Relative transcript abundances of EglLIM1 and Egl4CL1 were similar among the ten lines, while EglCAld5H1 levels exhibited more variation. This result indicates that the LIM domain transcription factor may control the expression of 4CL.

\section{Lignin characters and gene expression}

We measured $\mathrm{KL}$ content and the $\mathrm{S} / \mathrm{V}$ ratio in 6-month old plants of ten independent E. globulus lines. Figure 3 shows the relationships between transcript levels of the EglLIM1, Egl4CL1 and EglCAld5H1 genes, and KL

\section{b EgILIM1 vs S/V}
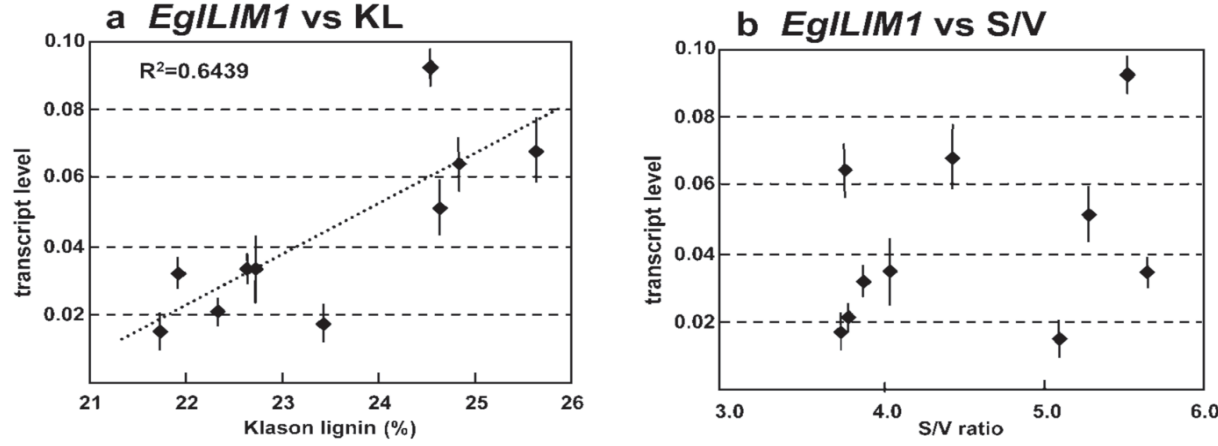

c Eg/4CL1 vs KL
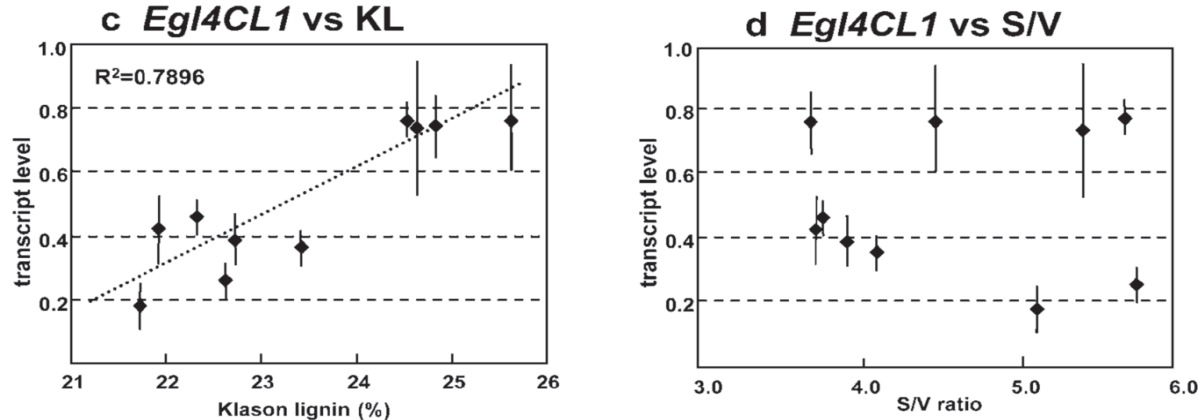

f EgICAId5H1 vs S/V
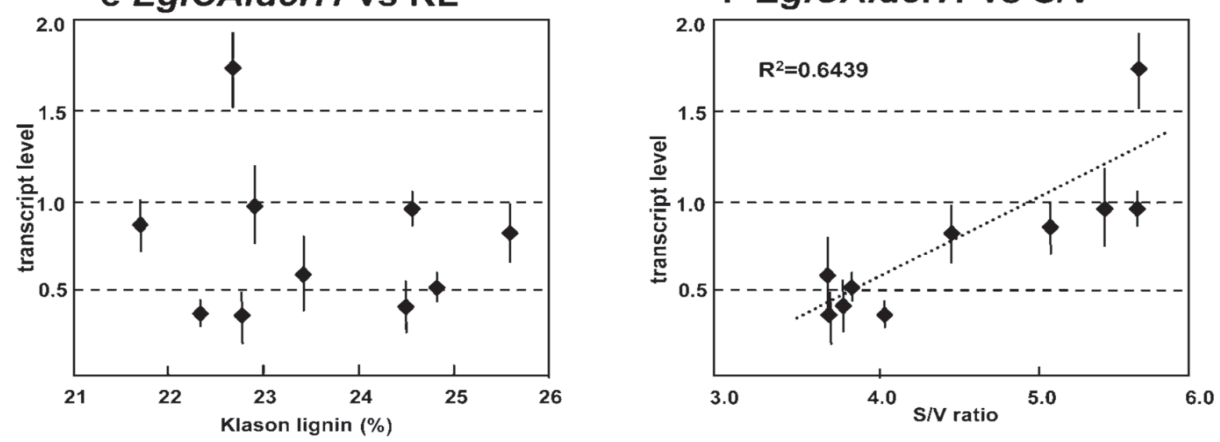

Figure 3. - Relationship between gene expression and wood quality parameters (KL content and $\mathrm{S} / \mathrm{V}$ ratio). Scatter plots showing transcript abundances relative to ubiquitin as determined by RT-qPCR. a: LIM transcript level vs. KL, b: $L I M$ transcript level vs S/V, c: $4 C L$ transcript level vs. KL, d: $4 C L$ transcript level vs. S/V, e: CAld5H transcript level vs.KL, f: CAld5H transcript level vs. S/V ratio. Error bars represent standard error values. 
contents or $\mathrm{S} / \mathrm{V}$ ratio. On an average, the plants contained $21.7-25.6 \% \mathrm{KL}$ and showed $\mathrm{S} / \mathrm{V}$ ratios between 3.7 and 5.8. A highly positive correlation $(\mathrm{p}<0.01)$ was observed between the transcript abundances of the LIM domain transcription factor, of 4CL1 genes and $\mathrm{KL}$ content of the plants (Figure $3 a, c$ ). A highly significant positive correlation $(\mathrm{p}<0.005)$ was observed between EglCAld5H1 expression and S/V ratio (Figure $3 f$ ).

\section{Gene structures of E. globulus three CesA genes}

To investigate the expression patterns of $E$. globulus CesA genes corresponding to EgraCesA1 to 3, the homologous CesA genes were isolated by screening an $E$. globulus cDNA library and their nucleotide sequences were determined. The putative amino acid sequences encoded by each gene (namely EglCesA1, EglCesA2 and EglCesA3) showed high similarities with the $E$. grandis predicted proteins $(97.9 \%, 97.5 \%$ and $98.7 \%$, respectively). Genomic DNA containing the EglCesA1 to 3 genes was isolated by PCR using specific primers (accession numbers, EglCesA1 cDNA, AB622343; EglCesA1 genomic, AB622342; EglCesA2 cDNA, AB623008; EglCesA2 genomic, AB623007; EglCesA3 cDNA, AB527048; EglCesA3 genomic, AB36817). The EglCesA1 and EglCesA3 genomic clones contained 13 exons and the EgCesA2 had 12 exons (Figure 4a). These exon numbers from ATG initiation codon were same as $E$. grandis CesA genes (Lu et al., 2008).

\section{Expression of CesA genes}

The transcript levels of EglCesA1 to 3 in each organ were assayed by RT-qPCR (Figure $4 b$ and $4 c$ ). All the three CesA genes showed similar level of transcript in the stems and shoot tips and exhibited high abundance in young stems. EglCesA1 to 3 mRNAs were not abundant in leaves. EglCesA1 and EglCesA3 showed high transcript abundances in the young stems and a relatively low abundances in middle and base stems (Figure $4 b, c)$.

We checked transcript abundances of EglCesA1 to 3 in ten independent $E$. globulus trees grown in a greenhouse. As before, total RNA was used from the upper region of stem and RT-qPCR was carried out using specific primers. EglCesA1 and EglCesA3 exhibited higher

a

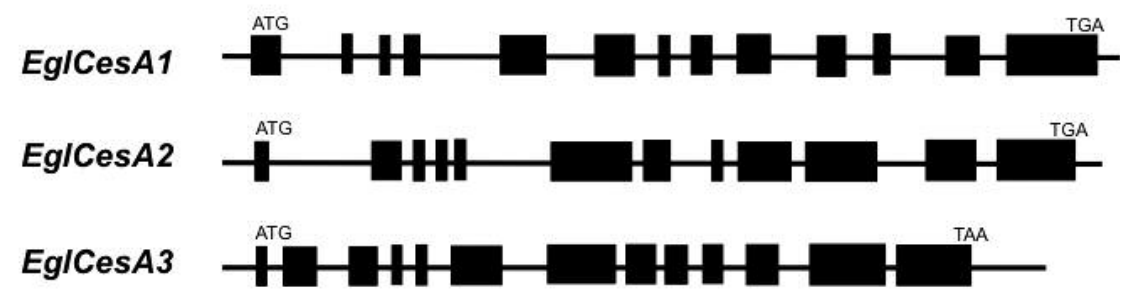

b

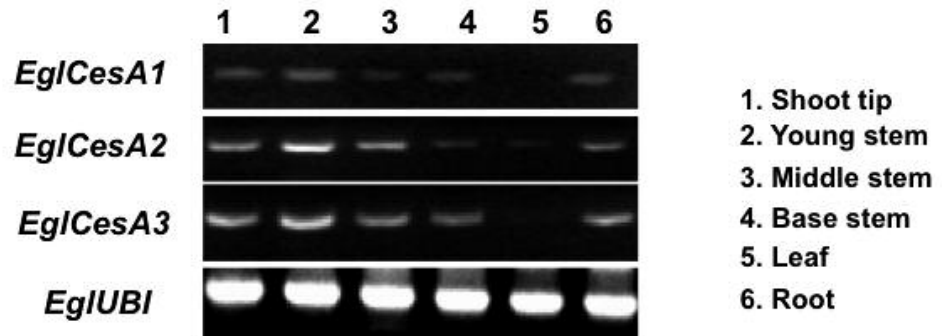

C

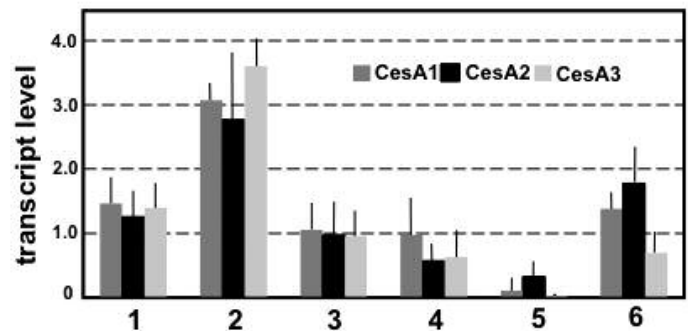

Figure 4. - Characterization of CesA genes in E. globulus.

a. Structure of the EglCesA1, EglCesA2 and EglCesA3 genes. Rectangular boxes show the exons; lines between exons indicate introns.

b. RT-PCR gel analysis of EglCesA1, EglCesA2 and EglCesA3 gene organ-specific expressions. Total RNA was extracted from different organs viz. shoot tip (1), young stem (2), middle stem (3), base stem (4), leaf (5) and root (6).

c. RT-qPCR analysis of EglCesA1, EglCesA2 and EglCesA3 gene expressions. The ubiquitin gene was used as an internal control. Each blot was replicated at least three times with fresh plant extracts. 


\section{a EgICesA1 to 3}

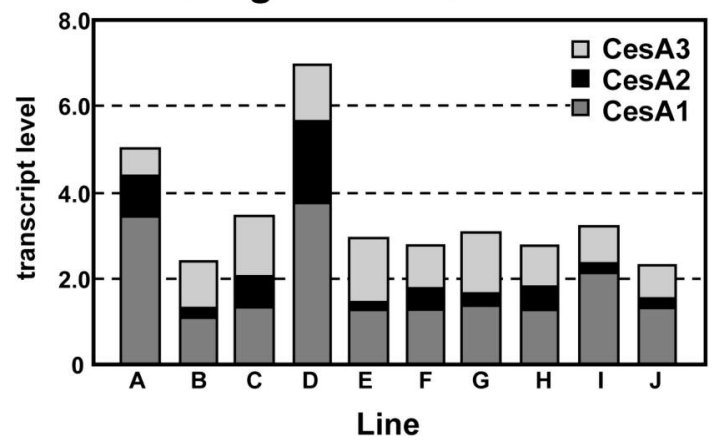

b $E g / C e s A s / E g / 4 C L$ vs $\mathrm{HC} / \mathrm{KL}$

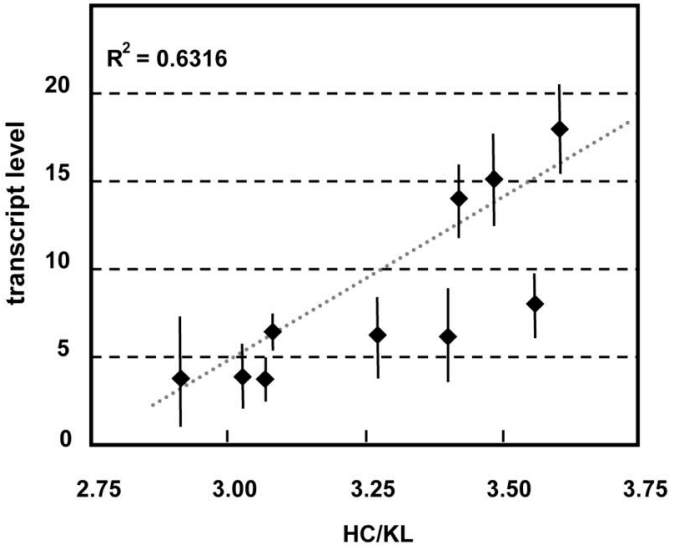

Figure 5. - Relationship between CesA gene expressions and wood quality parameters (HC/KL).

a. CesA1 to 3 gene transcript abundances of young stem in ten individual lines. The ubiquitin gene was used as an internal control. Each blot was replicated at least three times with fresh plant extracts and the error bars represent standard error values.

b. Relationship between gene expression ratio (sum of transcript abundances of EglCesA1, EglCesA2, and EglCesA3/ Egl4CL1) to HC/KL. Each data point represents the average of three replicates.

transcript abundances compared to EglCesA2 in most lines (Figure 5a).

\section{Analysis of wood character and chemical pulp yield}

We compared holocellulose (HC) content (cellulose and hemicellulose) with transcript abundances of the three CesAs. Transcript levels of the three genes showed no correlation with $\mathrm{HC}$ content $\left(\mathrm{R}^{2}<0.08\right)$. From the result that a 4CL transcript level strongly correlated to $\mathrm{KL}$ content, we investigated whether the ratio of $\mathrm{HC}$ content to $\mathrm{KL}$ content is related to the ratio of the sum of EglCesA1, EglCesA2 and EglCesA3 transcript levels to Egl4CL1 transcript level. The ratio (HC/KL) shows a highly positive correlation $(\mathrm{p}<0.01)$ to the ratio of the transcript abundances of CesA1 to 3 / 4CL (Figure $5 b$ ).

In order to understand the significance of the ratio of $\mathrm{HC} / \mathrm{KL}$, we investigated the correlation between $\mathrm{HC} / \mathrm{KL}$ and chemical kraft pulp (KP) yield. We harvested 7 to 10
$\mathrm{KP}$ yield vs $\mathrm{HC} / \mathrm{KL}$

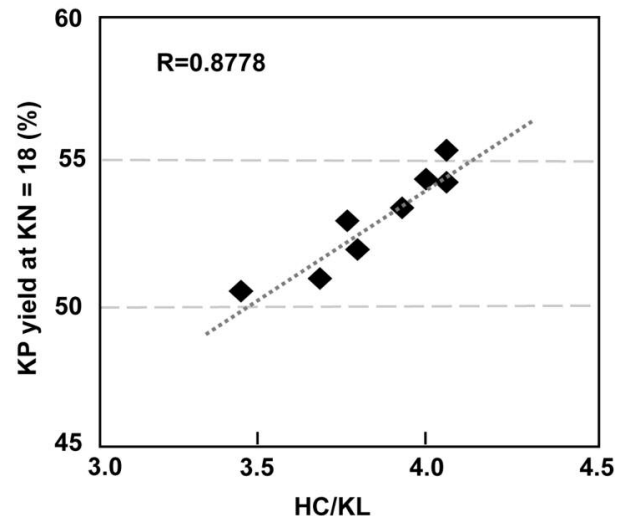

Figure 6. - Relationship between $\mathrm{KP}$ yields at $\mathrm{KN} 18$ and HC/KL ratios. Eight 7-10 year-old E. globulus trees were harvested in Western Australia and their wood chips were prepared and analyzed. Each data point represents the average of at least three replicates.

year-old $E$. globulus trees grown in Western Australia and imported the logs to Japan. Wood chips were prepared and cooked, and KP yields, $\mathrm{HC}$ and $\mathrm{KL}$ contents were simultaneously measured. A strong positive correlation between KP yields at kappa number (KN) 18 and ratios $\mathrm{HC}$ to $\mathrm{KL}$ was observed (Figure 6). The ratio of $\mathrm{HC} / \mathrm{KL}$ may be an indicator of fiber content because KP yield reflects the fiber volume that is chemically obtained.

\section{Discussion}

In order to improve wood-pulp production efficiency, efforts have been undertaken to modify the lignin content and composition using genetic engineering (VoNHOLME et al., 2008). In the past two decades, significant insights into the biosynthesis of lignin have been obtained by altering the expression of genes of the phenylpropanoid and monolignol biosynthetic pathways, and by studying the consequences of such manipulations for lignin content and composition, and for end-use properties (VONHOLME et al., 2008).

The amino acid sequence of EglLIM1 is identical to that of EcLIM1, suggesting that both proteins have the same physiological function. The EglLIM1 gene is expressed in $E$. globulus and exhibits a similar organspecific expression pattern as tobacco NtLIM1 and E. camaldulensis EcLIM1. (KAWAOKA et al., 2000; KAWAOKA et al., 2006). A high level of EglLIM1 transcript was observed in the entire stems (Figure 1b), which is in line with the idea that EglLIM1 is involved in lignification. The organ-specific expression pattern of the LIM resembles that of $4 \mathrm{CL}$, supporting the interpretation that $4 \mathrm{CL}$ is regulated by the LIM (KAWAOKA et al., 2000). Furthermore, the expression patterns of LIM and 4CL in basal stems of ten genetically independent $E$. globulus lines showed high similarity, whereas CAld5H showed a different pattern. This result corresponds well with the data from transgenic $E$. camaldulensis with downregu- 
lated EcLIM1, lending further support to the notion that the LIM protein regulates 4CL expression. The transcript abundances of 4CL and LIM were positively correlated with the $\mathrm{KL}$ content. This finding suggests that $4 \mathrm{CL}$ plays a role in the determination of lignin content which is well in line with previous results from transgenic tobacco and aspen plants (KAJITA et al., 1997; HU et al., 1999).

We also found that $\mathrm{CAld} 5 \mathrm{H}$ transcript abundances were positively correlated with the $\mathrm{S} / \mathrm{V}$ ratio (Figure $3 f$ ). Our results are consistent with previous findings in transgenic aspen plants with upregulated CAld5H (LI et al., 2003). In pulp production, $\mathrm{S}$ units are more easily removed than G units (RENCORET et al., 2007). Therefore, CAld5H is an important determinant of lignin quality.

The six cDNAs of CesA genes in $E$. grandis were isolated and characterized by RANIK and MYBURG (2006). EgraCesA1, EgraCesA2 and EgraCesA3 were abundantly expressed in cells producing secondary cell walls. EgraCesA3 was particularly strongly expressed in the xylem compared to the other five EgraCesA genes. Here, we isolated the EgraCesA1 to 3 homologs from $E$. globulus and found that their amino acid sequences were highly similar (by over $97 \%$ ) to the $E$. grandis proteins. Organ-specific expression patterns of EglCesA1 to 3 showed strong expression in shoot tips, stems and roots (Figure 4c). Similarly, the transcript of EgraCesA1 to 3 was particularly abundant in developing xylem (RANIK and Myburg, 2006; Lu et al., 2008). Higher transcript levels were observed in EglCesA1 to 3 in young stems at the primary cell wall formation stage (Figure $4 c$ ). Therefore, these three CesAs may be involved in primary cell wall formation. The value of a sum of transcript abundances of EglCesA1 to 3 divided Egl4CL1, correlated with a ratio of $\mathrm{HC}$ to $\mathrm{KL}$ content (Figure $5 b$ ). Individual CesA gene transcript level did not correlate well. In Eucalyptus wood, cellulose makes up approximately $50 \%$ of the dry weight and contributes to two thirds of the $\mathrm{HC}$ content. In our study, we found that the ratio of $\mathrm{HC}$ to $\mathrm{KL}$ content strongly correlated to the $\mathrm{KP}$ yield (Figure 6). KP contains $75-85 \%$ cellulose, 10-20\% hemicellulose and a few $\%$ of unextracted lignin, indicating the predominance of fiber material content (FARDIM and DURÁN, 2004). Therefore, the ratio HC/KL may very well reflect chemically-extracted fiber content of the wood.

Industrial interests provide a strong incentive for research on wood production. Here, we described how a regulatory gene (LIM) and a structural gene involved in lignin biosynthesis (4CL) affect lignin content in $E$. globulus. Our results show that the transcript abundances of CAld5H correlate with lignin composition. These results suggest a strategy to increase the final pulp yield. Pulp production requires the removal of lignin through harsh chemical treatment. Thus, woody plants with low lignin contents, S-rich lignin composition and high cellulose content are desirable for efficient pulp production. Eucalyptus plantation and breeding companies are working on effective selection of superior trees that have higher growth rates, basic density and KP yield. Our results indicate that lower transcript abundances of the LIM or 4CL genes could provide useful selection markers for lower lignin contents, while higher transcript abundances of CAld5H may positively affect lignin composition in Eucalyptus trees. A woody plant with higher CesA1 to 3 and lower 4CL gene expression may contain increased fiber content.

Recently, it has been reported that there are phenotypic differences between woody plants that are fieldgrown and greenhouse-grown. VOELKER et al. (2010) found physiological abnormalities in field-grown, lowlignin poplars. They also concluded that reductions in lignin content did not increase wood processability in such a way that they would benefit the production of liquid biofuels by fermentation. The results of this field study were different from the results of the greenhouse study we conducted with the transgenic poplar with antisense-4CL, which showed enhanced growth rate in a greenhouse (Hu et al., 1999). In the present study, we investigated correlations between the gene transcript abundances and wood qualities using woody plants grown in a greenhouse, where plants are mostly shielded from environmental stress. In the fields, woody plants are exposed to a number of abiotic stress factors such as low or high temperature, winds, drought, rain and snow. A greenhouse environment precludes such stress factors. As a future work, it would be interesting to see if similar results are obtained in the field. Overall, our results provide a strong foundation for manipulating candidate genes such as LIM domain transcription factor, $4 \mathrm{CL}, \mathrm{CAld} 5 \mathrm{H}$ and CesA towards the production of desirable wood qualities in an extremely important biomass species, Eucalyptus.

\section{Acknowledgements}

We thank TOSHIYUKI TAKANO of Kyoto University for technical support about wood property, JACQUELINE GRIMA-PETTENATI of UMR CNRS-UPS for providing gene information of E. gunnii, PHILIP UREN and KENJI YOSHINAGA of BTP for collecting wood samples.

\section{References}

Boerjan, W., J. RAlPH and M. BaUcher (2003): Lignin biosynthesis. Annu. Rev. Plant Biol. 54: 519-546.

Chiang, V. L. and M. FunAokA (1988): The dissolution and condensation reactions of guaiacyl and syringyl units in residual lignin during kraft deligninification of sweetgum. Holzforschaug. 44: 147-155.

Chomczynski, P. and N. SAcCHI (1987): Single-step method of RNA isolation by acid guanidinium thiocyanate-phenol-chloroform extraction. Annal. Biochem. 162: 156-159.

Endler, A. and S. Persson (2011): Cellulose synthases and synthesis in Arabidopsis. Mol. Plant, 4: 199-211.

EFFland, M. J. (1977): Modified procedure to determine acid insoluble lignin in wood and pulp. Tech. Assoc. Pulp Paper Ind. J. 60: 143-144.

FADIM, P. and N. DuRAN (2004): Retention of cellulose, xylan and lignin in kraft pulping of eucalyptus studied by multivariate data analysis: influences on physicochemical and mechanical properties of pulp. J. Braz. Chem. Soc. 15: 514-522. 
Gallo de Carvalro, M. C., D. G. Caldas, R. T. Carneiro, D. H. Moon, G. R. Salvatierra, L. M. Franceschini, A. De Andrade, P. A. Celedon, S. Oda and C. A. Labate (2008): SAGE transcript profiling of the juvenile cambial region of Eucalyptus grandis. Tree Physiol. 28: 905-919.

Goicoechea, M., E. Lacombe, S. Legay, S. Mihaljevic, P. Rech, A. Jauneau, C. Lapierre, B. Pollet, D. VerhaeGen, N. Chaubet-Giot and J. Grima-Pettenati (2005): EgMYB2, a new transcriptional activator from Eucalyptus xylem, regulates secondary cell wall formation and lignin biosynthesis. Plant J. 43: 553-567.

Hinchee, M., W. Rottmann, L. Mullinax, C. Zhang, S. Chang, M. Cunningham, L. Pearson and N. Nehra (2009) Short-rotation woody crops for bioenergy and biofuels applications. In Vitro Cell Biol. Plant. 45, 619-629.

Hu, W.-J., J. Lung, S. A. HARding, J. L. Popko, J. RAlPH, D. D. STokke, C.-J Tsai and V. L. ChiAng (1999): Repression of lignin biosynthesis promotes cellulose accumulation and growth in transgenic trees. Nat. Biotechnol. 17: 808-812.

Humphreys, J. M. and C. Chapple (2002): Rewriting the lignin roadmap. Curr. Opin. Plant Biol. 5: 224-229.

KaJiTA, S., S. HASHIMOTO, Y. TOMimURA, Y. KatayAmA and S. OMORI (1997): Structural characterization of modified lignin in transgenic tobacco plants in which the activity of 4-coumarate:coenzyme a ligase is depressed. Plant Physiol. 114: 871-879.

Kaothien, P., A. Kawaoka, H. Ebinuma, K. Yoshida and A. Shinmyo (2002): Ntlim1, a PAL-box binding factor, controls promoter activity of the horseradish woundinducible peroxidase gene. Plant Mol. Biol. 49: 591-599.

KaWAOKA, A., P. KaOThIEN, K. Yoshida, S. Endo, K. Yamada and H. EBINUMA (2000): Functional analysis of tobacco LIM protein Ntlim1 involved in lignin biosynthesis. Plant J. 22: 289-301.

KaWaOKa, A., K. Nanto, K. Ishit and H. Ebinuma (2006): Reduction of lignin content by suppression of expression of the LIM domain transcription factor in Eucalyptus camaldulensis. Silvae Genet. 55: 269-277.

Kubo, M., M. Udagawa, N. Nishikubo, G. Horiguchi, M. Yamagchi, J. Ito, T. Mimura, H. Fukuda and T. Demura (2005) Transcription switches for protoxylem and metaxylem vessel formation. Genes \& Develop. 19: 1855-1860.

Kumar, M., S. Thammanngowda, V. Bulone, V. Chiang, K. H. Han C. P. Joshi, S. D. Mansfield, E. Mellerowicz, B. Sundberg, T. TeERI and B. E. Ellis (2009): An update on the nomenclature for the cellulose synthase genes in Populus. Trends Plant Sci., 14: 248-254.

Li, L., Y. Zhou, X. Cheng, X. Sun, J. M. Marita, J. RalPh and V. L. CHIANG (2003): Combinatorial modification of multiple lignin traits in trees through multigene cotransformation. Proc. Natl. Acad. Sci. USA, 100: 4939-4944.

LI, X., J.-K. Weng and C. CHAPPELE (2008): Improvement of biomass through lignin modification. Plant J. 54: $569-581$.
LU, S., L. Li, X. YI, C. P. Joshi and V. L. ChIANG (2008): Differential expression of three eucalyptus secondary cell wall-related cellulose synthase genes in response to tension stress. J. Exp. Botany 59: 681-695.

Mitsuda, N., A. Iwase, H. Yamamoto, M. Yoshida, M. SEKI, K. SHINOZAKI and M. OHME-TAKAGI (2007): NAC Transcription Factors, NST1 and NST3, Are Key Regulators of the Formation of Secondary Walls in Woody Tissues of Arabidopsis. Plant Cell 19: 270-280.

Nagae, S., T. Takamura, T. Tanabe, A. Murakami, K. Murakami and M. TANaKa (1996): In vitro shoot development of Eucalyptus citriodora on Rockwool in the film culture vessel under $\mathrm{CO}_{2}$ enrichment. J. Forest Research 1: 227-230.

Pear, J. R., Y. Kawagoe, W. E. Schreckengost, D. P. DELMER and D. M. STALKER (1996): Higher plant contain homologs of the bacterial cesA genes encoding the catalytic subunit of cellulose synthase. Proc. Natl. Acad. Sci. USA 93: 12637-12642.

RANic, M. and A. A. MyBurg (2006): Six new cellulose synthase genes from Eucalyptus are associated with primary and secondary cell wall biosynthesis. Tree Physiol. 26: 545-556.

RENCORET, J., A. GutierRES and J. C. DEL RiO (2007): Lipid and lignin composition of woods from different eucalypt species. Holzforschung. 61: 165-174.

Rengel, D., H. San Clemente, F. Servant, N. Ladouce, E. Paux, P. Wincker, A. Couloux, P. Sivadon and J. GRima-Pettenati (2009): A new genomic resource dedicated to wood formation in Eucalyptus. BMC Plant Biol. 9: 36-49.

Rogers L. A. and M. M. CAMPBell (2004) The genetic control of lignin deposition during plant growth and development. New phytologist. 164: 17-30.

SARKNEN, K. V. (1971): Lignins, pp. 19-42. In: Occurrences, Formation, Structure and Reaction, edited by K. V. SARKNen and C. H. Ludwig, Wiley Interscience, New York.

Voelker, S. L., B. Lachenbruch, F. C. Meinzer, M. JourDes, C. Ki, A. M. Patten, L. B. Davin, N. G. Lewis, G. A. Tuskan, L. Gunter, S. R. Decker, M. J. SElig, R. Sykes, M. E. Himmel, P. Kitin, O. Sheychenko and S. H. STRAUSS (2010): Antisense-down-regulation of 4CL expression alters lignification, tree growth, and saccharification potential of field-grown poplar. Plant Phisyol. 154: 874-886.

Vonholme, R., K. Morreel, J. Ralph and W. Boerjan (2008): Lignin engineering. Curr. Opi. Plant Biol. 11: $278-285$.

Weng, J. K., L. Xu, J. Stout and C. C. Chapple (2008): Independent origins of syringyl lignin in vascular plants. Pro. Natl. Acad. Sci. USA, 105: 7887-7892.

Wise, L. E., M. MuRPhy and A. A. D'AdDIECo (1946): Chlorite holocellulose, its fractionation and bearing on summative wood analysis and on studies on hemicellulose. Paper Trade J., 122: 35-43.

Herausgeber: Johann Heinrich von Thünen-Institut. Bundesforschungsinstitut für Ländliche Räume, Wald und Fischerei. Schriftleitung: Institut für Forstgenetik, Sieker Landstrasse 2, D-22927 Grosshansdorf Verlag: J. D. Sauerländer's Verlag, Berliner Strasse 46, D-63619 Bad Orb Anzeigenverwaltung: J. D. Sauerländer's Verlag, Bad Orb

Gesamtherstellung: PPPP Norbert Wege e.K., Gladenbach — Printed in Germany. 